\title{
Recent results on 2HDM charged Higgs boson searches in CMS
}

\author{
Santeri Laurila ${ }^{* t}$ \\ Helsinki Institute of Physics \\ E-mail: santeri.laurila@helsinki.fi
}

The results of two recent CMS physics analyses searching for charged Higgs bosons $\left(\mathrm{H}^{ \pm}\right)$are presented. They are based on data from proton-proton collisions at the CERN LHC, recorded by the CMS experiment. In the first analysis, a charged Higgs boson with a mass from 90 to $150 \mathrm{GeV}$ decaying to a c quark and a b quark is searched for in electron+jets and muon+jets final states of top quark pair events. The search is performed using data collected by CMS in 2012 at $8 \mathrm{TeV}$, corresponding to a total integrated luminosity of $19.7 \mathrm{fb}^{-1}$. The signal is extracted by reconstructing the invariant mass of two jets, one of which is identified as a b-jet. No $\mathrm{H}^{ \pm}$signal is observed and upper limits of $1.1-0.4 \%$ are set at $95 \%$ confidence level on the branching fraction for $\mathrm{t} \rightarrow \mathrm{H}^{ \pm} \mathrm{b}$ for the charged Higgs boson in the mass range of 90-150 GeV, assuming $B\left(\mathrm{H}^{ \pm} \rightarrow \mathrm{cb}\right)=1$. In the second analysis, a charged Higgs boson with a mass from $80 \mathrm{GeV}$ to $3 \mathrm{TeV}$ decaying to a tau lepton and a neutrino is searched for in the fully hadronic final state of top quark pair events. The search is performed using data collected by CMS in 2016 at $13 \mathrm{TeV}$, corresponding to a total integrated luminosity of $12.9 \mathrm{fb}^{-1}$. Signal is extracted by reconstructing the transverse mass of the hadronically decaying tau lepton and the missing transverse momentum. No $\mathrm{H}^{ \pm}$signal is observed and upper limits of $2.8-0.3 \%$ are set at $95 \%$ confidence level on $B\left(\mathrm{t} \rightarrow \mathrm{bH}^{ \pm}\right) \times B\left(\mathrm{H}^{ \pm} \rightarrow \tau^{ \pm} v\right)$ for the charged Higgs boson mass range of $80-160 \mathrm{GeV}$, while upper limits of 1.8-0.004 pb are set at $95 \%$ confidence level on $\sigma\left(\mathrm{pp} \rightarrow \mathrm{tbH}^{ \pm}\right) \times B\left(\mathrm{H}^{ \pm} \rightarrow \tau^{ \pm} v\right)$ for the charged Higgs boson mass ranging from $180 \mathrm{GeV}$ to $3 \mathrm{TeV}$.

Prospects for Charged Higgs Discovery at Colliders

3-6 October 2016

Uppsala, Sweden

\footnotetext{
* Speaker.

${ }^{\dagger}$ For CMS Collaboration.
} 


\section{Introduction}

In many BSM scenarios there are several Higgs bosons, some of which can be charged. The two Higgs doublet models (2HDM) [1,2] predict five Higgs bosons: the light and heavy CP-even Higgs bosons $\mathrm{h}$ and $\mathrm{H}$, the CP-odd Higgs boson $\mathrm{A}$, and two charged Higgs bosons $\mathrm{H}^{ \pm}$. Light charged Higgs bosons $\left(\mathrm{H}^{+}\right.$mass smaller than the top quark mass) can be produced in top quark decays, while heavy charged Higgs bosons can be produced in association with a top quark: $\mathrm{pp} \rightarrow$ $\overline{\mathrm{t} b \mathrm{H}^{+}}$. (Throughout the text, charge conjugated processes are implied.)

Previously, charged Higgs bosons have been directly searched for in $\mathrm{H}^{+} \rightarrow \tau \nu$ [3-7] and $\mathrm{H}^{+} \rightarrow \mathrm{cs}$ [8-11] decays at hadron collider experiments. Here, two recent CMS analyses searching for 2HDM charged Higgs bosons are presented. The first CMS result on $\mathrm{H}^{+} \rightarrow \mathrm{c} \bar{b}$ search channel is based on $19.7 \mathrm{fb}^{-1}$ of data from the Run 1 of CERN LHC, collected at a center-of-mass energy of $\sqrt{s}=8 \mathrm{TeV}$. The analysis is documented in Ref. [12]. Also the first $13 \mathrm{TeV}$ result by CMS on the $\mathrm{H}^{+} \rightarrow \tau \nu$ channel is presented, based on $12.9 \mathrm{fb}^{-1}$ of data collected in 2016 at $\sqrt{s}=13 \mathrm{TeV}$. The analysis is documented in Ref. [13]. Examples of Feynman diagrams corresponding to the two search channels are shown in Fig. 1.
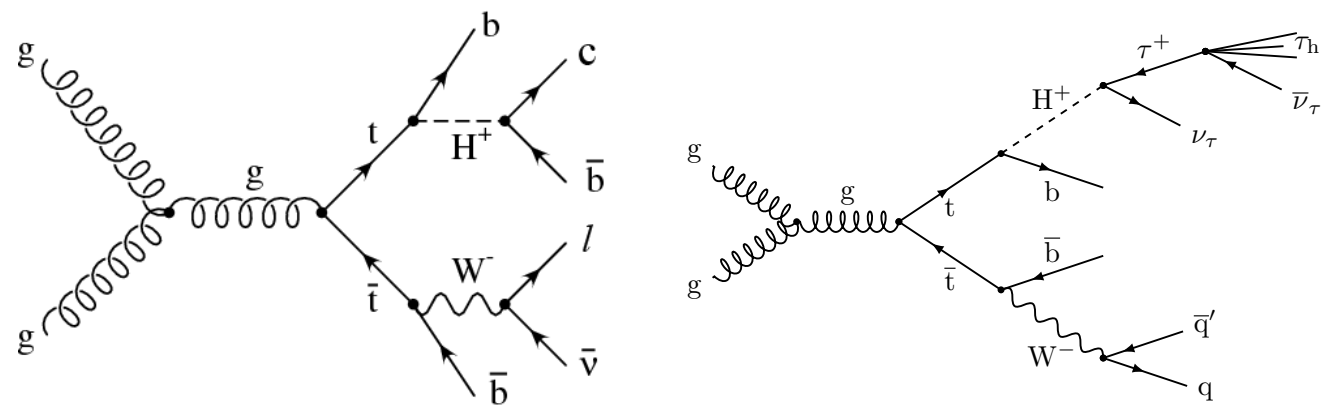

Figure 1: Examples of charged Higgs boson Feynman diagrams for the light $\mathrm{H}^{+}$produced in top quark decay and decaying as $\mathrm{t} \rightarrow \mathrm{H}^{+} \mathrm{b}$ (left) or $\mathrm{H}^{+} \rightarrow \tau \nu$ (right).

\section{CMS detector and event reconstruction}

The central feature of the CMS detector is a superconducting solenoid with an internal radius of $3 \mathrm{~m}$, providing a magnetic field of $3.8 \mathrm{~T}$. A more detailed description of the CMS detector, as well as definitions of the coordinate system used, can be found in Ref. [14].

Final state objects used in the analyses presented here are reconstructed using the standard CMS particle flow (PF) algorithm [15, 16]. The anti- $k_{T}$ algorithm [17] with a distance parameter $R=0.5$ (at $8 \mathrm{TeV}$ ) or $R=0.4$ (at $13 \mathrm{TeV}$ ) is used in jet clustering. Jets originating from b quarks are identified (b-tagged) using the combined secondary vertex (CSV) algorithm [18]. In this text, "b-jet" is used to refer to any b-tagged jet. Hadronically decaying tau leptons $\left(\tau_{\mathrm{h}}\right)$ are reconstructed using the hadron-plus-strips algorithm [19]. The $\tau_{\mathrm{h}}$ candidates that are compatible with muons or electrons are rejected. Jets are suppressed by requiring the $\tau_{\mathrm{h}}$ candidate to be isolated.

The missing transverse momentum vector $\vec{E}_{T}^{\text {miss }}$ is defined as the projection on the plane perpendicular to the beams of the negative vector sum of the momenta of all reconstructed particles in an event. Its magnitude is referred to as missing transverse energy $E_{T}^{\text {miss }}$. 


\section{3. $\mathrm{H}^{+} \rightarrow \mathrm{c} \bar{b}$ search}

In this section, the first CMS analysis looking for the $\mathrm{H}^{+} \rightarrow \mathrm{c} \bar{b}$ decay is presented. The $\mathrm{H}^{+} \rightarrow \mathrm{c} \overline{\mathrm{b}}$ branching fraction is enhanced in the type-Y 2HDM model [20,21], leading to $\mathrm{H}^{+}$in top decays as $\mathrm{t} \rightarrow \mathrm{H}^{+} \mathrm{b} \rightarrow \mathrm{c} \overline{\mathrm{b}} \mathrm{b}$. Thus $\mathrm{t} \overline{\mathrm{t}}$ production can lead to events with one top quark decaying to a charged Higgs boson and subsequently giving three jets (two of which are b-jets), while the other top quark decays leptonically: $\mathrm{t} \rightarrow \mathrm{W}^{-} \mathrm{b}$ and $\mathrm{W}^{-} \rightarrow \ell \bar{v}$. As shown in Fig. 1, this gives a final state with three b-jets, compared with only two from the semi-leptonic SM t⿱t decays.

This analysis searches for a charged Higgs boson by looking for a (non-W) peak in the dijet mass spectrum reconstructed from the two jets that are most likely to originate from the $\mathrm{H}^{+}$. The search is performed in two categories: events with two b-tagged jets and events with at least three btagged jets. Figure 2 shows the dijet mass distribution for the $\mathrm{H}^{+}$and $\mathrm{W}$ decays for both categories.
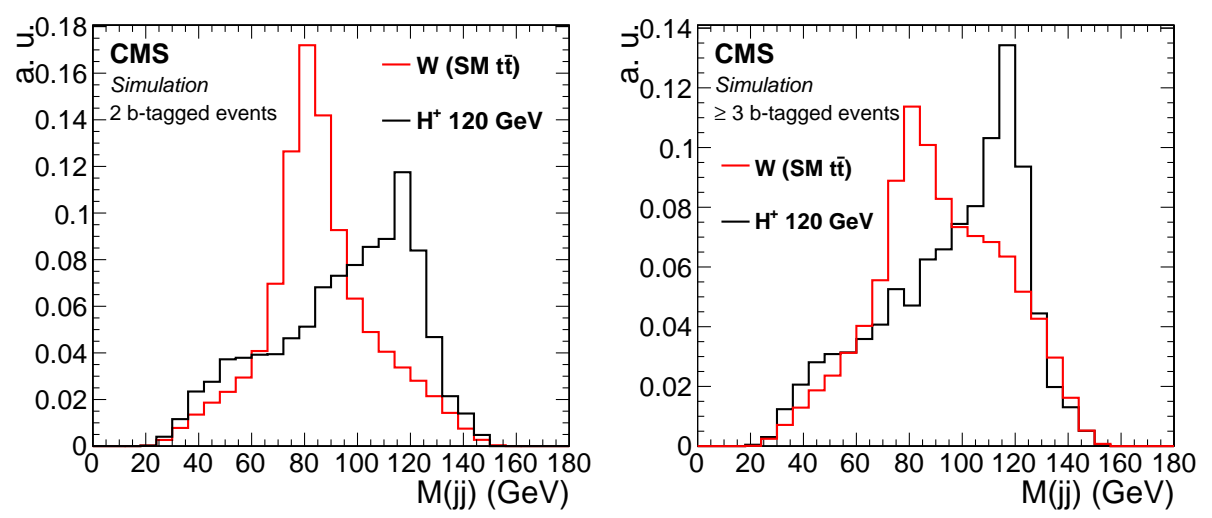

Figure 2: Simulated dijet mass distribution for the $\mathrm{W}$ boson in the $\mathrm{SM} t \overline{\mathrm{t}}$ events and for the $\mathrm{H}^{+}$in the signal events, for event categories with two b-tagged jets (left) and with more than two b-tagged jets (right).

The charged Higgs boson signal events are simulated using PYTHIA 6.4 [22] and the background samples are generated with MADGRAPH 5.1.3.30 [23], POWHEG v1.0 r1380 [24] and PYTHIA 6.4. Parton showering and hadronization are simulated using PYTHIA 6.4, and the full simulation of the CMS detector is performed using GEANT4 [25]. Pileup is simulated by mixing the simulated hard events with multiple minimum bias events and weighting the simulated events such that the pileup distribution matches that of the real data.

\subsection{Event selection}

Signal events like the one shown in Fig. 1 contain one prompt lepton, one neutrino, and four quarks, three of which are b quarks. Therefore the final state observed in the detector will contain either a muon or an electron, at least four jets and missing transverse energy.

Single lepton triggers are used for both the muon channel $\left(p_{T}>24 \mathrm{GeV},|\eta|<2.1\right)$ and the electron channel ( $p_{T}>27 \mathrm{GeV},|\eta|<2.5$ ). The electrons (muons) are required to be well reconstructed and isolated, to have $p_{T}>30 \mathrm{GeV}(26 \mathrm{GeV}),|\eta|<2.5$ (2.1) and to be consistent with originating from the primary vertex. Events with more than one lepton are rejected. 
Jets are required to have $p_{T}>30 \mathrm{GeV}$ and $|\eta|<2.4$. Since the analysis looks for events which include a neutrino in the final state, $E_{T}^{\text {miss }}>20 \mathrm{GeV}$ is required. In the identification of b-jets, the medium working point (misidentification rate of $1 \%$ ) is used.

The analysis uses four separate signal event categories, depending on the lepton flavour (electron or muon) and the number of b-tagged jets (two or more). Thus the categories are: electron+jets events with two b-jets, electron+jets with at least three b-jets, muon+jets events with two b-jets and muon+jets with at least three b-jets.

\subsection{Background estimations}

Three types of backgrounds are considered in this analysis: backgrounds from the top quark ( $\bar{t} \bar{t}$ and single top quark decays), backgrounds not associated with the top quark (non-top backgrounds), and QCD multijet events with a jet misidentified as a lepton.

In the categories with two b-jets, the estimation of the top quark and non-top backgrounds is performed using simulation. In the categories with at least thre b-jets, there are two complications. Firstly, events from SM t $\bar{t}$ decays have two components: $\bar{t}+j$ ets (with one of non-b jets mistagged as $a b$-jet) and $\bar{t} \bar{t}+b \bar{b}$ events. Simulation of the latter process is known to give too few events [26,27], so the simulated events are corrected using a scale factor measured from a control region with high $\mathrm{t} \overline{\mathrm{t}}+\mathrm{b} \overline{\mathrm{b}}$ purity, defined by two isolated leptons of opposite charge, large $E_{T}^{\text {miss }}$, and three b-tagged jets. In the control region the data/MC ratio is found to be $1.23 \pm 0.10$. Secondly, as non-top backgrounds suffer from low statistics in these categories, the estimation is done by using events with two b-jets and correcting for the different tagging efficiencies with a scale factor.

For the background from multijet events, a data driven technique is used. An "anti-isolated" sample is obtained using signal-like events in the data, except the relative isolation of a lepton is taken to be looser. Background events estimated from simulated samples are processed through this anti-isolated selection and subtracted from the data. To correct for the difference in normalization of events between the signal selection and the anti-isolated selection, a scale factor is applied to the anti-isolated sample. This scale factor is measured using a control region orthogonal to the signal region and to the anti-isolated region, defined identically to the signal region except that $E_{T}^{\text {miss }}<20 \mathrm{GeV}$. The scale factor is obtained using events with two b-jets, and applied to the QCD multijet background estimation in all event categories. The same strategy to estimate the multijet background has been used previously in Ref. [10].

\subsection{Parton specific corrections to jets}

As a good dijet mass resolution is essential for the sensitivity of this analysis, additional parton-specific jet energy corrections are introduced in order to make the jet energy match better to the energy of the primary quark of a specific flavour (b, c, or $\mathrm{u} / \mathrm{d} / \mathrm{s})$. The parton specific corrections are derived by comparing the matched parton $p_{T}$ with the reconstructed jet $p_{T}$.

The assignment of jets to specific quarks and the selection of the jet pair to be associated with a $\mathrm{H}^{+}$boson decay are performed using a kinematic fitter. While constraining the invariant masses of the leptonic $\mathrm{W}$ and both the leptonic and hadronic top quarks to the true values used in simulation, the fitter tries to find the jet to parton assignment with the minimal $\chi^{2}$ by varying the $p_{T}$ values of the leading four jets, the lepton, and the non-clustered jet energy of an event. 

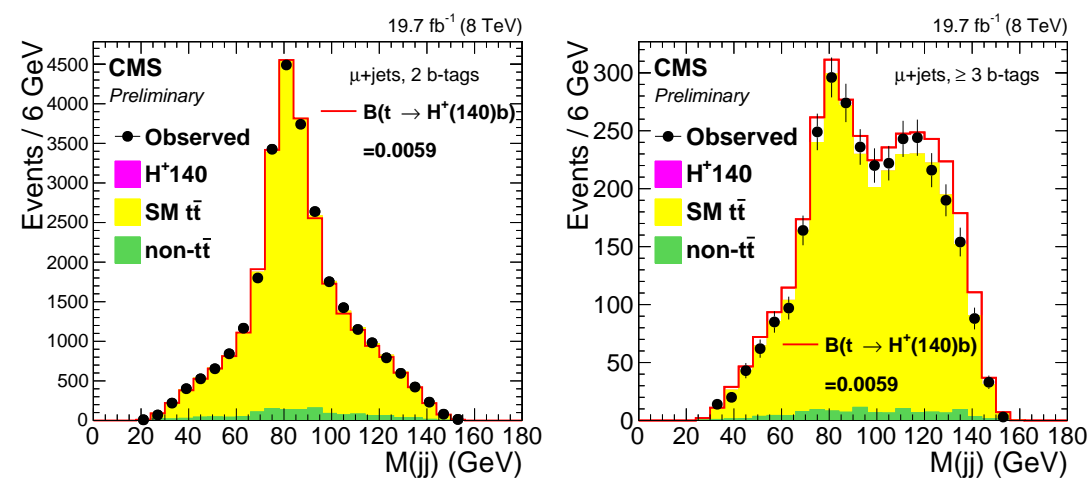

Figure 3: Dijet mass distribution in the $\mu$ +jets events with two b-jets fitted on the observed data by a Maximum Likelihood fit. A simulated signal normalized to this branching fraction is overlaid.

The matching between a parton and a jet is done using a cone of $\Delta R<0.3$. Only the four leading jets are assumed to originate from the tree level partons and used for the t⿱t $\mathrm{t}$ reconstruction. All sources of systematic uncertainties considered in the analysis are discussed in Ref. [12].

\subsection{Results}

The observed data in the four signal categories are fitted using the Maximum Likelihood method to determine the branching fraction $B\left(\mathrm{t} \rightarrow \mathrm{H}^{+} \mathrm{b}\right)$, assuming $B\left(\mathrm{H}^{+} \rightarrow \mathrm{c} \overline{\mathrm{b}}\right)=1$, using binned dijet mass templates for the background and the signal, normalized by the best fit values shown in Fig. 3 (for the $\mu+$ jets channel).

The best-fit values for $B\left(\mathrm{t} \rightarrow \mathrm{H}^{+} \mathrm{b}\right)$ for all Higgs boson mass hypotheses are consistent with the background-only hypothesis. Combining both electron and muon channels, an upper limit from 0.4 to $1.1 \%$ at $95 \%$ confidence level (CL) is obtained. The limit is shown in Fig. 4.

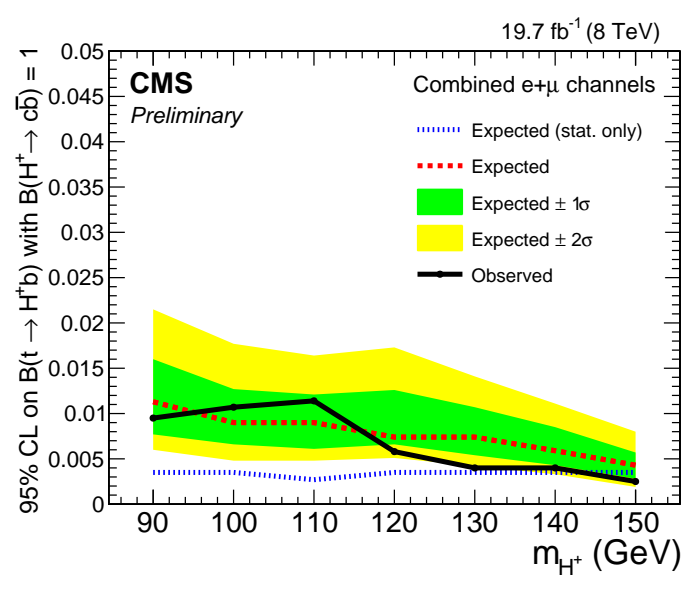

Figure 4: The observed upper limit on $B\left(\mathrm{t} \rightarrow \mathrm{H}^{+} \mathrm{b}\right)$ at $95 \% \mathrm{CL}$ assuming $B\left(\mathrm{H}^{+} \rightarrow \mathrm{c} \bar{b}\right)=1$ (black line). The green (yellow) uncertainty bands correspond to one (two) standard deviations of the expected limit (red line). 


\section{4. $\mathrm{H}^{+} \rightarrow \tau v$ search}

In this section, the first CMS Run 2 analysis looking for $\mathrm{H}^{+} \rightarrow \tau v$ decay is presented. This analysis focuses on the fully hadronic final state containing a tau jet from the $\mathrm{H}^{+}$decay, hadronic jets (including b-jets) and missing transverse energy due to neutrinos from the $\mathrm{H}^{+}$decay. The benefit of the fully hadronic final state is that the transverse mass, defined as [28]

$$
m_{\mathrm{T}}=\sqrt{2 p_{T}\left(\tau_{\mathrm{h}}\right) E_{T}^{\mathrm{miss}}\left(1-\cos \Delta \phi\left(\vec{E}_{T}^{\mathrm{miss}}, \tau_{\mathrm{h}}\right)\right)}
$$

can be reconstructed from the hadronic tau $\tau_{\mathrm{h}}$ and $\vec{E}_{T}^{\text {miss }}$ and used to extract the signal.

The signal samples are generated with MadGraph5_aMC@NLO [29] for $\mathrm{H}^{+}$masses ranging from $80 \mathrm{GeV}$ to $3 \mathrm{TeV}$. A custom NLO 2HDM UFO model [30] is used for the hard process. All background processes containg a genuine tau lepton are estimated from simulation: these include the top pair production ( $\overline{\mathrm{t}} \mathrm{t})$ at next-to-next-to-leading order (NNLO), single top production at nextto-leading order (NLO) [31-34], Drell-Yan production [29], W+jets production at leading order (LO) in QCD with up to 4 associated partons [35], and diboson production [29,36].

Parton showering and hadronization, simulation of the CMS detector and pileup are performed as discussed in Section 3. Background from events with mis-identified tau (fake tau background) is estimated using a data-driven method.

\subsection{Event selection}

As the fully hadronic final state is common for the light $\mathrm{H}^{+}$(produced in top quark decay) and the heavy $\mathrm{H}^{+}$(produced in association with a top quark), the same analysis is used for both cases. Only the selection thresholds in tau $p_{T}$ and $E_{T}^{\text {miss }}$ selections are optimized separately.

The online trigger requires a hadronic tau with $p_{T}>50 \mathrm{GeV}$ and $|\eta|<2.1$, containing a leading charged hadron with $p_{T}>30 \mathrm{GeV}$, and missing transverse energy of $E_{T}^{\text {miss }}>90 \mathrm{GeV}$. Events are first required to contain a well reconstructed primary vertex. In offline selection, the events are required to have tau $p_{T}>50(60) \mathrm{GeV}$ and $E_{T}^{\text {miss }}>90$ (100) $\mathrm{GeV}$ for the light (heavy) $\mathrm{H}^{+}$ analysis. Events containing any isolated electron or muon are rejected.

Three hadronic jets with $p_{T}>30 \mathrm{GeV}$ and $|\eta|<4.7$ are required, and at least one of these is required to be a b-tagged jet with $|\eta|<2.5$. Moreover, an event variable $R_{\mathrm{bb}}^{\min }$, defined as [37]

$$
R_{\mathrm{bb}}^{\min }=\min _{j \in j_{1} . . j_{3}} \sqrt{\Delta \phi\left(E_{T}^{\mathrm{miss}}, j\right)^{2}+\left(\pi-\Delta \phi\left(\tau_{\mathrm{h}}, E_{T}^{\mathrm{miss}}\right)\right)^{2}}
$$

where $j_{1} . . j_{3}$ are the three leading jets in the event, is used to reject multijet events where the $\vec{E}_{T}^{\text {miss }}$ and $\tau_{\mathrm{h}}$ are in back-to-back configuration, by requiring $R_{\mathrm{bb}}^{\min }>40^{\circ}$, as shown in Fig. 5 .

\subsection{Background estimation}

The main backgrounds are an irreducible background from standard model EWK+t的 events with a genuine tau, QCD multijet events with a hadronic jet mis-identified as a tau jet (QCD fake tau), and $\mathrm{EWK}+\mathrm{t} \overline{\mathrm{t}}$ events with a jet or a lepton mis-identified as a hadronic tau $(\mathrm{EWK}+\mathrm{t} \overline{\mathrm{t}}$ fake tau). The EWK processes include W+jets, Drell-Yan and diboson production. The QCD multijet 

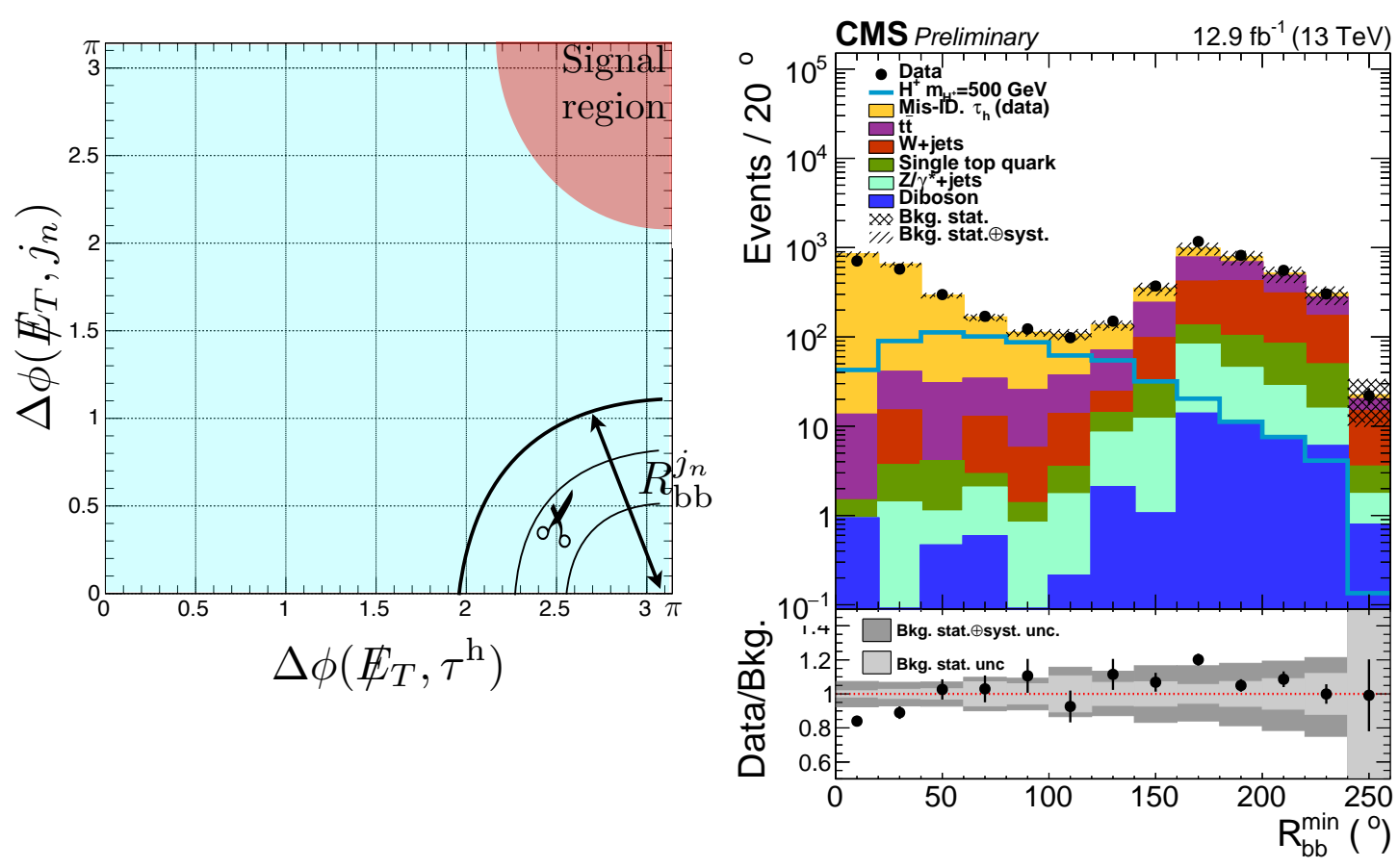

Figure 5: Signal region and the $R_{\mathrm{bb}}^{\min }$ cut shown in the $\Delta \phi\left(\tau_{\mathrm{h}}, E_{T}^{\text {miss }}\right)-\Delta \phi\left(\tau_{\mathrm{h}}, j_{n}\right)$ plane (left), and the distribution of $R_{\mathrm{bb}}^{\mathrm{min}}$ after all previous selections (right).

and EWK+tt fake tau backgrounds are estimated using a data-driven method, while the EWK+tt genuine tau backgrounds are estimated from simulation. Corrections are applied to the simulated events to better model the data.

The fake tau background with misidentified tau leptons is measured from a control region, defined by inverting the isolation requirement on the hadronic tau leptons (inverted selection). The data and the simulated genuine tau background samples are processed through the inverted selection, and the genuine tau background contribution is subtracted from the data. The resulting $E_{T}^{\text {miss }}$ distribution, estimated in the control region, has been checked to have a shape similar to the $E_{T}^{\text {miss }}$ distribution of the fake tau background in the signal region. The shape of the $E_{T}^{\text {miss }}$ distribution in the control region and the shape of the $E_{T}^{\text {miss }}$ distribution of genuine tau events in the signal region are used to determine the fraction of fake tau events in the data in the signal region, using a maximum likelihood fit on $E_{T}^{\text {miss }}$ templates. Based on the calculated fraction, the fake tau events from the control region are weighted to correspond to the estimated event yield of the fake tau events in the signal region. To minimize the effect of correlations between tau $p_{T}$ and $E_{T}^{\text {miss }}$, the measurements and fits are done separately in tau $p_{T}$ bins.

The dominant sources of systematic uncertainties are related to the $\tau_{\mathrm{h}}$, the jet and the $E_{T}^{\text {miss }}$ energy scales and the normalization of the fake tau background. A more detailed description of the systematic uncertainties is given in Ref. [13]. The sensitivity of the light $\mathrm{H}^{+}$analysis is mostly constrained by these systematic uncertainties, while for the heavy $\mathrm{H}^{+}$analysis the limited amount of data is still the most important constraint. 

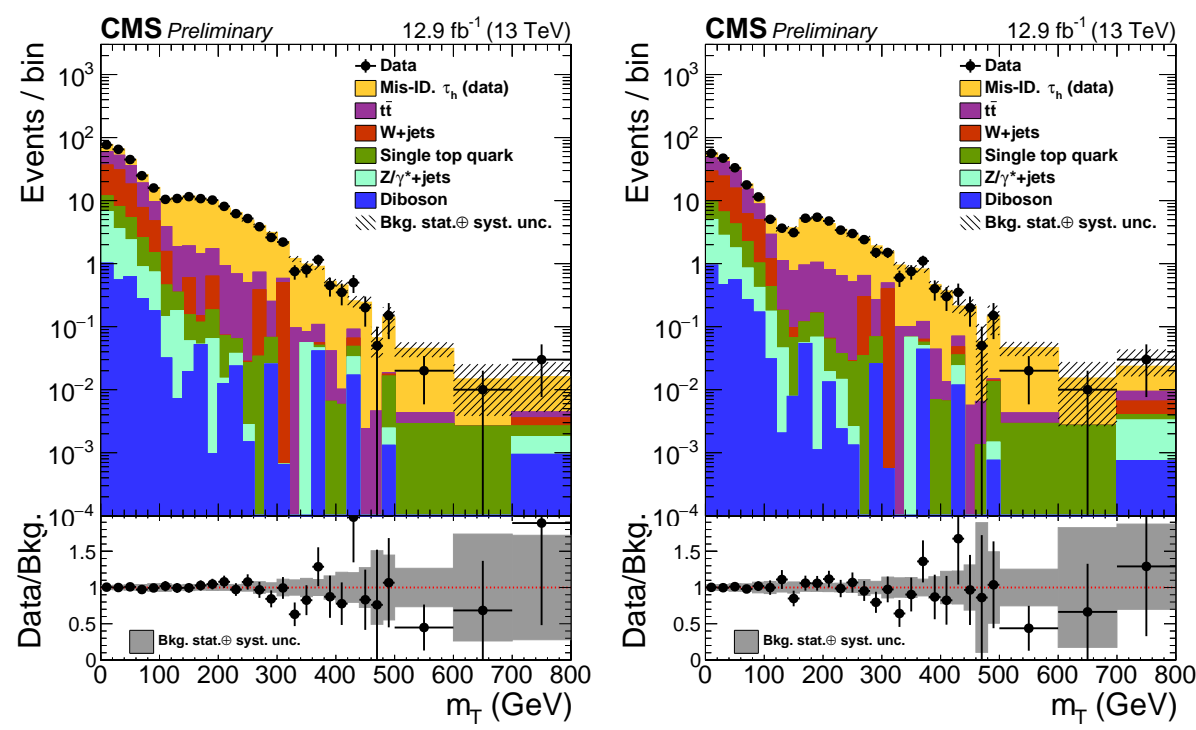

Figure 6: The transverse mass $m_{\mathrm{T}}$ distribution observed in data, compared to the fitted background predictions in the light $\mathrm{H}^{+}$(left) and the heavy $\mathrm{H}^{+}$(right) searches.

\subsection{Results}

The signal is extracted by performing a binned Maximum Likelihood fit to the transverse mass distribution, shown in Fig. 6. The fit is performed both in the background-only and signal+background scenarios, and the 95\% CL upper limits are set on the $\mathrm{H}^{+}$production using the $\mathrm{CL}_{\mathrm{s}}$ criterion $[38,39]$ on the likelihood ratio test-statistics $[40,41]$.

The resulting upper limits on $B\left(\mathrm{t} \rightarrow \mathrm{bH}^{ \pm}\right) \times B\left(\mathrm{H}^{ \pm} \rightarrow \tau^{ \pm} v\right)$ are shown in Fig. 7 (left) for the mass range of $80-160 \mathrm{GeV}$, while the upper limits on the product of production cross section $\sigma\left(\mathrm{pp} \rightarrow \mathrm{tbH}^{ \pm}\right)$and $B\left(\mathrm{H}^{ \pm} \rightarrow \tau^{ \pm} v\right)$ are shown in Fig. 7 (right) for the mass ranging from $180 \mathrm{GeV}$ to $3 \mathrm{TeV}$. Both limits are compatible with the background-only hypothesis.
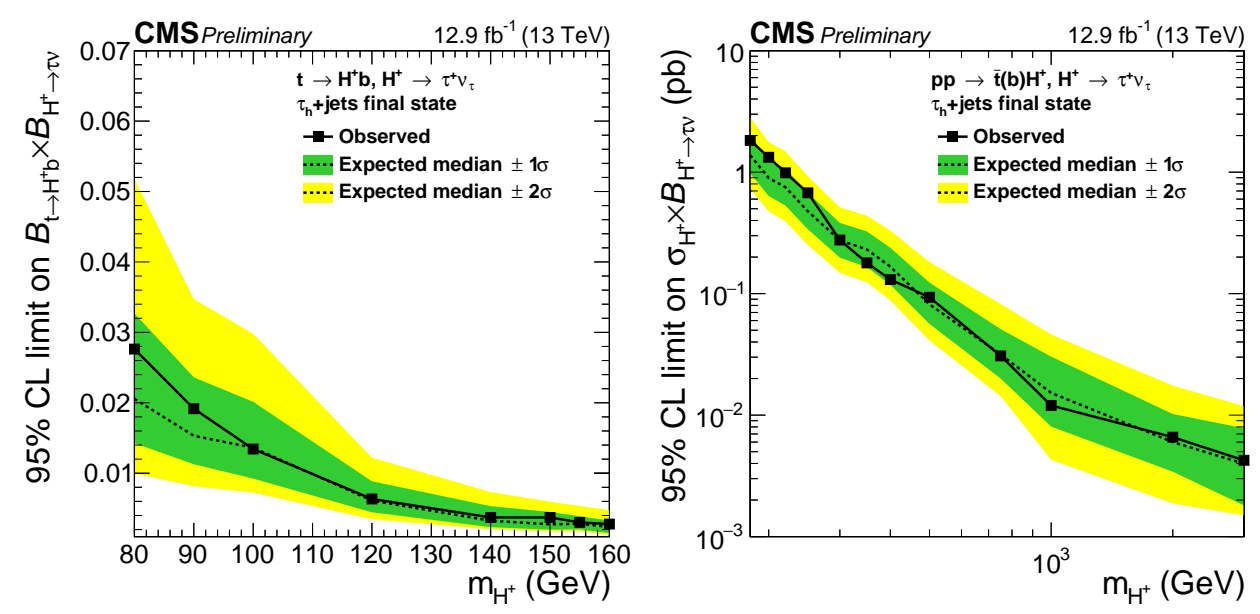

Figure 7: The observed $95 \% \mathrm{CL}$ upper limits on $B\left(\mathrm{t} \rightarrow \mathrm{bH}^{ \pm}\right) \times B\left(\mathrm{H}^{ \pm} \rightarrow \tau^{ \pm} v\right)$ (left) and $\sigma(\mathrm{pp} \rightarrow$ $\left.\mathrm{tbH}^{ \pm}\right) \times B\left(\mathrm{H}^{ \pm} \rightarrow \tau^{ \pm} v\right)$ (right) and the corresponding expected limits. The green (yellow) uncertainty bands correspond to one (two) standard deviations of the expected limits. 


\section{References}

[1] J. F. Gunion, S. Dawson, H. E. Haber and G. L. Kane, The Higgs hunter's guide, vol. 80. Brookhaven Nat. Lab., Upton, NY, 1989.

[2] G. C. Branco, P. M. Ferreira, L. Lavoura, M. N. Rebelo, M. Sher and J. P. Silva, Theory and phenomenology of two-Higgs-doublet models, Phys. Rept. 516 (2012) 1-102, [1106. 0034 ].

[3] CDF collaboration, A. Abulencia et al., Search for charged Higgs bosons from top quark decays in $p \bar{p}$ collisions at $\sqrt{s}=1.96-T e V$. , Phys. Rev. Lett. 96 (2006) 042003, [hep-ex/ 0510065 ].

[4] D0 collaboration, V. M. Abazov et al., Search for charged Higgs bosons in decays of top quarks, Phys. Rev. D80 (2009) 051107, [0 906.5326 ].

[5] CMS collaboration, S. Chatrchyan et al., Search for a light charged Higgs boson in top quark decays in pp collisions at $\sqrt{s}=7 \mathrm{TeV}$, JHEP 07 (2012) 143, [1205.5736].

[6] ATLAS collaboration, G. Aad et al., Search for charged Higgs bosons decaying via $H^{ \pm} \rightarrow \tau^{ \pm} v$ in fully hadronic final states using pp collision data at $\sqrt{s}=8 \mathrm{TeV}$ with the ATLAS detector, JHEP 03 (2015) 088, [1412.6663].

[7] ATLAS collaboration, G. Aad et al., Search for charged Higgs bosons decaying via $H^{+} \rightarrow \tau v$ in top quark pair events using pp collision data at $\sqrt{s}=7$ TeV with the ATLAS detector, JHEP 06 (2012) 039, [1204.2760].

[8] CDF collaboration, T. Aaltonen et al., Search for charged Higgs bosons in decays of top quarks in $p$ anti-p collisions at $s^{* *}(1 / 2)=1.96$ TeV, Phys. Rev. Lett. 103 (2009) 101803, [0 907.1269 ].

[9] D0 collaboration, V. M. Abazov et al., Search for Charged Higgs Bosons in Top Quark Decays, Phys. Lett. B682 (2009) 278-286, [0 908 . 1811].

[10] CMS collaboration, V. Khachatryan et al., Search for a light charged Higgs boson decaying to $\mathrm{c} \overline{\mathrm{s}}$ in pp collisions at $\sqrt{s}=8 \mathrm{TeV}$, JHEP 12 (2015) 178, [1510.04252].

[11] ATLAS collaboration, G. Aad et al., Search for a light charged Higgs boson in the decay channel $H^{+} \rightarrow c \bar{s}$ in $t \bar{t}$ events using pp collisions at $\sqrt{s}=7$ TeV with the ATLAS detector, Eur. Phys. J. C73 (2013) 2465, [1302 . 3694].

[12] CMS collaboration, C. Collaboration, Search for Charged Higgs boson to $\mathrm{c} \overline{\mathrm{b}}$ in lepton+jets channel using top quark pair events, .

[13] CMS collaboration, C. Collaboration, Search for charged Higgs bosons with the $\mathrm{H}^{ \pm} \rightarrow \tau^{ \pm} v_{\tau}$ decay channel in the fully hadronic final state at $\sqrt{s}=13 \mathrm{TeV}$, .

[14] CMS collaboration, S. Chatrchyan et al., The CMS experiment at the CERN LHC, JINST 3 (2008) S08004.

[15] CMS collaboration, CMS, Particle-flow event reconstruction in CMS and performance for jets, taus, and $E_{T}^{\text {miss }}$, CMS Physics Analysis Summary CMS-PAS-PFT-09-001, 2009.

[16] CMS collaboration, CMS, Commissioning of the Particle-flow Event Reconstruction with the first LHC collisions recorded in the CMS detector, CMS Physics Analysis Summary CMS-PAS-PFT-10-001, 2010.

[17] M. Cacciari, G. P. Salam and G. Soyez, The Anti-k(t) jet clustering algorithm, JHEP 0804 (2008) 063, [0802.1189].

[18] CMS collaboration, S. Chatrchyan et al., Identification of b-quark jets with the CMS experiment, JINST 8 (2013) P04013, [1211. 4462 ].

[19] CMS collaboration, V. Khachatryan et al., Reconstruction and identification of $\tau$ lepton decays to hadrons and $v_{\tau}$ at CMS, JINST 11 (2016) P01019, [1510.07488].

[20] M. Aoki, S. Kanemura, K. Tsumura and K. Yagyu, Models of Yukawa interaction in the two Higgs doublet model, and their collider phenomenology, Phys. Rev. D80 (2009) 015017, [0 902 . 4 665]. 
[21] H. E. Logan and D. MacLennan, Charged Higgs phenomenology in the flipped two Higgs doublet model, Phys. Rev. D81 (2010) 075016, [1002 .4916].

[22] T. Sjostrand, S. Mrenna and P. Z. Skands, PYTHIA 6.4 Physics and Manual, JHEP 05 (2006) 026, [hep-ph/0603175].

[23] J. Alwall, M. Herquet, F. Maltoni, O. Mattelaer and T. Stelzer, MadGraph 5 : Going Beyond, JHEP 06 (2011) 128, [1106.0522].

[24] S. Alioli, P. Nason, C. Oleari and E. Re, A general framework for implementing NLO calculations in shower Monte Carlo programs: the POWHEG BOX, JHEP 06 (2010) 043, [1 002 . 2581].

[25] J. Allison et al., Geant4 developments and applications, IEEE Trans. Nucl. Sci. 53 (2006) 270.

[26] CMS collaboration, V. Khachatryan et al., Measurement of $\mathrm{t} \overline{\mathrm{t}}$ production with additional jet activity, including $\mathrm{b}$ quark jets, in the dilepton decay channel using pp collisions at $\sqrt{s}=8 \mathrm{TeV}$, Eur. Phys. J. C76 (2016) 379, [1510.03072].

[27] CMS collaboration, V. Khachatryan et al., Measurement of the cross section ratio $\sigma_{\mathrm{t} \overline{\mathrm{t}} \overline{\mathrm{b}}} / \sigma_{\mathrm{t} \mathrm{tjj}}$ in $p p$ collisions at $\sqrt{s}=8 \mathrm{TeV}$, Phys. Lett. B746 (2015) 132-153, [1411.5621].

[28] Particle Data Group collaboration, K. A. Olive et al., Review of Particle Physics, Chin. Phys. C38 (2014) 090001.

[29] J. Alwall, R. Frederix, S. Frixione, V. Hirschi, F. Maltoni, O. Mattelaer et al., The automated computation of tree-level and next-to-leading order differential cross sections, and their matching to parton shower simulations, JHEP 07 (2014) 079, [1405.0301].

[30] M. Ubiali and C. Degrange. Private communication.

[31] S. Alioli, P. Nason, C. Oleari and E. Re, NLO single-top production matched with shower in POWHEG: s-and t-channel contributions, JHEP 09 (2009) 111, [0 907 . 4076 ].

[32] S. Alioli, P. Nason, C. Oleari and E. Re, NLO single-top production matched with shower in POWHEG: s- and t-channel contributions, JHEP 09 (2009) 111, [0 907 . 4 076 ].

[33] E. Re, Single-top Wt-channel production matched with parton showers using the POWHEG method, Eur. Phys. J. C71 (2011) 1547, [1009.2450].

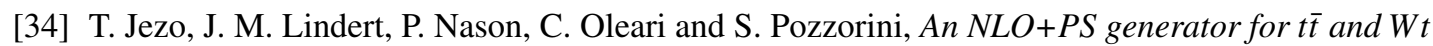
production and decay including non-resonant and interference effects, 1607.04538.

[35] J. Alwall, M. Herquet, F. Maltoni, O. Mattelaer and T. Stelzer, MadGraph 5 : Going Beyond, JHEP 06 (2011) 128, [1106.0522].

[36] P. Nason and G. Zanderighi, $W^{+} W^{-}, W Z$ and ZZ production in the POWHEG-BOX-V2, Eur. Phys. J. C74 (2014) 2702, [1311.1365].

[37] CMS collaboration, V. Khachatryan et al., Search for a charged Higgs boson in pp collisions at $\sqrt{s}=8 \mathrm{TeV}$, JHEP 11 (2015) 018, [1508.07774].

[38] A. L. Read, Presentation of search results: the cls technique, J. Phys. G28 (2002) 2693.

[39] T. Junk, Confidence level computation for combining searches with small statistics, Nucl.Instrum.Meth. A434 (1999) 435-443, [hep-ex/9902006].

[40] Procedure for the LHC Higgs boson search combination in summer 2011, Tech. Rep. ATL-PHYS-PUB-2011-011, CERN, Geneva, Aug, 2011.

[41] L. Moneta, K. Belasco, K. S. Cranmer, A. Lazzaro, D. Piparo, G. Schott et al., The RooStats Project, in $13^{\text {th }}$ International Workshop on Advanced Computing and Analysis Techniques in Physics Research (ACAT2010), SISSA, 2010. 1009.1003. 